\title{
RECHT
}

\section{Bürokratiekosten per Gesetz}

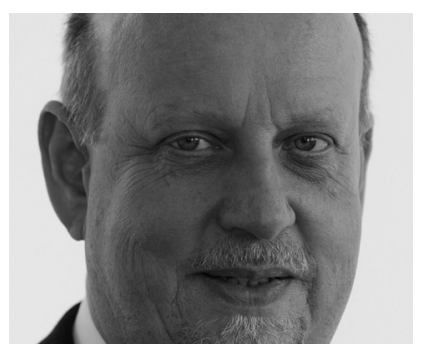

VON STEFAN SCHICK

Prof. Dr. Stefan Schick ist als

Rechtsanwalt und Fachanwalt für Steuerrecht in Stuttgart tätig. Er ist seit langem Mitglied des Beirats der Zeitschrift SOZIALwirtschaft und des Informationsdienstes SOZIALwirtschaft aktuell.

E-Mail

Stefan.Schick@reithschick.de
Ein nicht unerheblicher Anteil der Bürokratiekosten in Deutschland dürfte durch komplizierte rechtliche und steuerliche Regelungen verursacht sein. Das Bestreben, alle denkbaren Einzelfälle in Gesetzen berücksichtigen zu wollen, führt zu kaum mehr durchschaubaren Bestimmungen, die auch Experten überfordern, wie Beispiele aus dem Sozialbereich zeigen.

Welcher Rechtsanwalt oder Steuerberater kennt sie nicht - die Frage, ob man Aussagen zu rechtlichen und steuerlichen Fragen nicht auch in verständlichem Deutsch treffen kann. Denn wenn sich eine komplizierte Rechtssprache und oft komplexe Gesetzesregelungen begegnen, wird die Rechtsfindung - nicht nur für den Laien - zum Problem. Einigen prägnanten Beispielen und der Suche nach den Ursachen ist dieser Beitrag gewidmet.

\section{Problembereich: Die Entstehung komplizierter Regelungen}

Erkennt der Gesetzgeber die Notwendigkeit, bestimmte Sachverhalte gesetzlich zu regeln, so ist das Gesetz bei seiner erstmaligen Verkündung in der Regel noch - relativ - überschaubar und verständlich. Häufig ergibt sich aber in der Folgezeit, dass bestimmte Fälle nicht oder nicht in dem Sinne geregelt wurden, den sich der Gesetzgeber vorgestellt hat. Hier stellt sich aber schon die Frage "Wer ist eigentlich der Gesetzgeber, wer für die Gesetze zuständig? « Häufig geht dann die Initiative nicht von der Politik, sondern von der Ministerialbürokratie aus: Das Gesetz wird »nachgebessert ", dadurch aber auch komplizierter.

Ein sehr schönes Beispiel für eine solche, am Einzelfall orientierte Nachbesserung bildet die Entstehung der derzeitigen Regelung in $\$ 12$ Abs. 2 Nr. 8a) UStG. Nach dieser Bestimmung sind Umsätze steuerbegünstigter Körperschaften, soweit sie nicht durch steuerpflichtige wirtschaftliche Geschäftsbetriebe erbracht werden, dem ermäßigten Steuersatz von 7 Prozent unterworfen. Dies betrifft die gemeinnützigkeitsrechtlichen Sphären der Vermögensverwaltung und des Zweckbetriebs, jeweils soweit die Umsätze nicht von der Umsatzsteuer befreit sind.
Anlass für die Neuregelung war der folgende Fall: Eine gemeinnützige Organisation hatte eine pfiffige Geschäftsidee. Sie gründete nämlich ein Integrationsunternehmen, bestehend aus einem behinderten und einem nicht behinderten Mitarbeiter. Das Integrationsunternehmen erfüllte die gemeinnützigkeitsrechtlichen Anforderungen eines steuerbegünstigten Zweckbetriebs nach $\$ 68 \mathrm{Nr} .3 \mathrm{c}$ ) AO. Dies hatte nach $\mathbb{} 2$ Abs. 2 Nr. 8a) UStG zur Folge, dass seine Umsätze dem ermäßigten Steuersatz von 7 Prozent unterlagen. Im Gegenzug dazu war das Integrationsunternehmen im Hinblick auf die von ihm eingekauften Leistungen vorsteuerabzugsberechtigt.

Zweck des Integrationsunternehmens war der Handel mit EDV-Hardware. Veränderungen an den eingekauften Geräten wurden vom Integrationsunternehmen nicht vorgenommen. Folge der Gestaltung: Die Hardware wurde - belastet mit dem vollen Umsatzsteuersatz von damals noch 16 Prozent - eingekauft, die Vorsteuer wurde abgezogen, und die Ware wurde an nicht vorsteuerabzugsberechtigte Kunden (Wohlfahrtsverbände, aber auch die öffentliche Hand) - dann mit einer nur 7-prozentigen Umsatzsteuer belastet - weiterverkauft. Der Steuervorteil wurde teilweise weitergegeben und bildete damit einen Wettbewerbsvorteil gegenüber gewerblichen Händlern. Im Übrigen erwirtschaftete die gemeinnützige Organisation so einen Gewinn.

Reaktion der Finanzverwaltung: Sie verlangte - zunächst in einer Verwaltungsregelung, dann im Rahmen einer Gesetzesänderung -, dass der ermäßigte Steuersatz dann nicht gelten solle, wenn der Zweck des Zweckbetriebs der Erzielung zusätzlicher Einnahmen dient.

Reaktion der Wohlfahrtsverbände: Diese bemängelten, dass mit einer solchen Regelung alle Leistungen der Zweckbetriebe dem vollen Umsatzsteuersatz unterliegen würden, da sich Zweckbetriebe immer - zumindest auch - durch die Erzielung zusätzlicher Einnahmen finanzieren.

Reaktion des Gesetzgebers: Er kombinierte beide Anliegen. $\$ 12$ Abs. 2 Nr. 8a) UStG hat jetzt folgenden Wortlaut: »Die Steuer ermäßigt sich auf 7 Prozent für die 
folgenden Umsätze: die Leistungen der Körperschaften, die ausschließlich und unmittelbar gemeinnützige, mildtätige oder kirchliche Zwecke verfolgen $(\mathbb{S} \mathbb{S} 51$ bis 68 der Abgabenordnung). Dies gilt nicht für Leistungen, die im Rahmen eines wirtschaftlichen Geschäftsbetriebs ausgeführt werden. Für Leistungen, die im Rahmen eines Zweckbetriebs ausgeführt werden, gilt Satz 1 nur, wenn der Zweckbetrieb nicht in erster Linie der Erzielung zusätzlicher Einnahmen durch die Ausführung von Umsätzen dient, die in unmittelbarem Wettbewerb mit dem allgemeinen Steuersatz unterliegenden Leistungen anderer Unternehmer ausgeführt werden, oder wenn die Körperschaft mit diesen Leistungen ihrer in den $\mathbb{S} \int 66$ bis 68 der Abgabenordnung bezeichneten Zweckbetriebe ihre steuerbegünstigten satzungsmäßigen Zwecke selbst verwirklicht. «

\section{Quintessenz dieser Gesetzesänderung:}

- Es lag ein Missbrauchsfall vor, der bereits von der ganz allgemeinen Regelung des $\mathbb{4} 42 \mathrm{AO}$ erfasst war. Es wäre denkbar gewesen, diese Fallgestaltung umsatzsteuerlich so zu behandeln, als wäre die Leistung nicht im Rahmen eines steuerbegünstigten Zweckbetriebs erbracht worden. Eine Einzelfallregelung wäre dann eigentlich nicht erforderlich gewesen.

- Der Versuch, auch den letzten Einzelfall durch die gesetzliche Regelung ausdrücklich zu erfassen, führt dazu, dass es Ausnahmen von der Ausnahme gibt das Gesetz wurde komplizierter, unverständlicher. Wer sein Recht sucht, benötigt häufig einen - guten - Berater. Es verbleibt die Frage, ob komplizierte Gesetze, die alle denkbaren Fälle möglichst gerecht regeln wollen, aber so kompliziert werden, dass sie kaum noch verständlich sind, wirklich (sach-) gerecht sind.

\section{Problembereich: Nicht aufeinander abgestimmte Regelungen und Begrifflichkeiten in unterschiedlichen Gesetzen}

Viele Juristen haben (noch) die Vision von der Einheit der Rechtsordnung. Sie gehen davon aus oder hoffen zumindest, dass zum Einen gleichlautende Begriffe in verschiedenen Zusammenhängen dieselbe Bedeutung haben und dass Regelungen in unterschiedlichen Gesetzen aufeinander abgestimmt sind. Doch das ist ein Trugschluss!

Beispiel: Nach $\mathbb{2}$ Abs. 3 UStG sind die juristischen Personen des öffentlichen Rechts nur im Rahmen ihrer Betriebe ge- werblicher Art im Sinne des Körperschaftsteuerrechts und ihrer land- und forstwirtschaftlichen Betriebe gewerblich oder beruflich tätig. Trotz der direkten Verweisung auf das Körperschaftsteuerrecht wird jedoch mittlerweile der Betrieb gewerblicher Art im Umsatzsteuerrecht (aufgrund EU-rechtlicher Vorgaben) anders definiert als im Körperschaftsteuerrecht!

Die Tatsache, dass »der Gesetzgeber « Einzelfragen und Einzelprobleme grundsätzlich rechtsgebietsbezogen abar- der zu beurteilende Sachverhalt für die spätere steuerliche Behandlung abgeklärt wird, so dass sich der Aufwand im Veranlagungsverfahren reduziert. Dennoch sind die Finanzämter im Hinblick auf ihre angespannte Personalsituation häufig überfordert.

Welche Folgen dann ein Antrag auf Erteilung einer verbindlichen Auskunft haben kann, zeigt der folgende Fall: Eine steuerbegünstigte Körperschaft hat sich 2009 entschlossen, eine Umstrukturierung durchzuführen. Sie stellt im Dezember 2009 einen

\section{"Der Versuch, auch den letzten Einzelfall sge- rechtı regeln zu wollen, führt zu unverständli- chen Gesetzestexten "}

beitet und regelt, führt dazu, dass parallele Regelungen entstehen, die nicht aufeinander abgestimmt sind.

Beispiel: Die Qualitätssicherung von Pflegeeinrichtungen wird sowohl im SGB XI als auch im Heimrecht geregelt. Außerdem führen Pflegeeinrichtungen häufig zusätzlich Qualitätsprüfungen durch und lassen sich zertifizieren. Dies führt $\mathrm{zu}$ Mehrfachprüfungen durch unterschiedliche Prüfungsorganisationen auf der Grundlage unterschiedlicher Kriterien.

Hier hat allerdings der Gesetzgeber die Notwendigkeit erkannt, trotz unterschiedlicher Prüfungsansätze möglichst Doppelund Mehrfachprüfungen zu vermeiden. Der Weg zur definitiven Umsetzung dürfte aber noch weit sein.

\section{Problembereich: Der Umgang mit komplizierten Regelungen am Beispiel des Steuerrechts}

Eine wesentliche Hilfe im Steuerrecht bildet die sogenannte »verbindliche Auskunft «. Ein Steuerpflichtiger, der beispielsweise eine Umgestaltung vornimmt, kann bis 2006 nur im Rahmen eines Verwaltungserlasses, seither nach $\mathbb{8} 89$ Abs. 2 AO auf gesetzlicher Grundlage - die steuerliche Behandlung der beabsichtigten Gestaltung im Vorfeld mit der Finanzverwaltung verbindlich abstimmen. Seit der gesetzlichen Neuregelung fallen dafür auch Gebühren an, die sich nach dem Wert richten. Kann der Wert nicht festgestellt werden, wird eine Zeitgebühr - pro angefangener halber Stunde Arbeitszeit des Finanzbeamten 50 Euro - berechnet.

Diese Abklärung hat nicht nur für den Steuerpflichtigen den Vorteil, dass er die steuerlichen Folgen einer Gestaltung kennt und sich darauf einstellen kann. Die verbindliche Auskunft führt auch dazu, dass
Antrag auf verbindliche Auskunft und erhält zwei Wochen später ein Schreiben des Finanzamts, in dem - unter zweimaliger Berufung auf einen Antrag vom September 2009 (den es nie gab - es wurde ein anderer Vorgang als Vorlage verwendet) - die Erteilung der verbindlichen Auskunft (vorläufig) abgelehnt wurde. Die vorgetragenen Rechtfragen seien eindeutig; die Voraussetzungen einer verbindlichen Auskunft seien nicht erfüllt. Weitergehende Ausführungen im Januar 2010 hatten zur Folge, dass das Finanzamt mit der Prüfung begann und Mitte Februar 2010 mitteilte, die Rechtsfragen seien so schwierig, dass eine Bearbeitung vor Mitte März nicht möglich sei. Der Antrag ist Mitte Mai 2010 noch immer nicht bearbeitet. Er habe die personellen Ressourcen dafür nicht, so der zuständige Sachgebietsleiter. Die Gebühren für die Bearbeitung - es ist von mehreren Tausend Euro auszugehen - könne er nicht für Neueinstellungen oder für den Einsatz zusätzlichen Personals einsetzen.

\section{Ansätze für eine Problemlösung im Sinne der Entbürokratisierung}

Für Gesetzgebungsverfahren lassen sich die folgenden Appelle zusammenfassen:

- Die Zusammenfassung der wesentlichen Grundsätze im »allgemeinen Teil « - für das Steuerrecht in den $\mathbb{S} 1$ bis $50 \mathrm{AO}$ erhöht die Übersichtlichkeit und entschlackt Gesetze. Vor einer detaillierten Ergänzung von Einzelregelungen sollte konsequenter geprüft werden, ob der Einzelfall nicht bereits unter Heranziehung allgemeiner Regelungen befriedigend gelöst werden kann.

- Es muss nicht zwingend alles ausdrücklich gesetzlich geregelt werden; man sollte mehr auf die Gesetzesauslegung vertrauen. 
- Es sollte abgewogen werden, ob nicht im Sinne einer Entbürokratisierung gegebenenfalls auch Regelungslücken in Kauf genommen werden sollten, anstelle die letzte theoretisch noch denkbare Fallgestaltung zu regeln. Ein kompliziertes Gesetz wird nicht dadurch gerechter, dass es alles zu regeln versucht (was ohnehin kaum gelingen wird). Es führt dadurch zur Ungerechtigkeit, dass es kaum noch anwendbar und beherrschbar ist: Selbst derjenige, der daraus Rechte ableiten könnte, kann sie nicht mehr geltend machen, weil er das Gesetz und damit die für ihn günstige Rechtslage nicht mehr versteht.

- Gleiche Begrifflichkeiten und Regelungsinhalte in unterschiedlichen Rechtsgebieten sollten harmonisiert werden.

Am Ende bleibt aber auch die Hoffnung und Erwartung, dass der Staat die bestehenden gesetzlichen Regelungen und Verwaltungsvorschriften bereinigt:

- Mit Kabinettsbeschluss vom 21. April 2010 hat die Bundesregierung ein Gesetz auf den Weg gebracht, mit dem mehr als 80 Gesetze und Verordnungen aufgehoben werden. "Wir brauchen so viel Recht wie nötig, nicht so viel Recht wie möglich «, so Bundesjustizministerin Leutheusser-Schnarrenberger. Bei 1.716 Bundesgesetzen und 2.644 Rechtsverordnungen sei die Pflege des Bundesrechts eine wichtige Aufgabe, so die $\mathrm{Mi}$ nisterin (http://www.bmj.bund.de/enid).

- Parallel dazu legt die Finanzverwaltung im Rahmen einer Positivliste fest, welche Schreiben des Bundesfinanzministeriums, die bis zum 31. Dezember 2009 ergangen sind, weitergelten (BMF-Schreiben vom 23. April 2010, Gz.: IV A 6-O 1000/10095, DOK 1010/0197416).

Übrigens: Manchmal gerät der Zweck einer Steuer nachträglich (absichtlich?) in Vergessenheit, so wie bei der 1902 zur Finanzierung der kaiserlichen Flotte eingeführten Schaumweinsteuer. Ob es noch mehr solche Beispiele gibt?

\section{Akzeptanz von Hartz IV?}

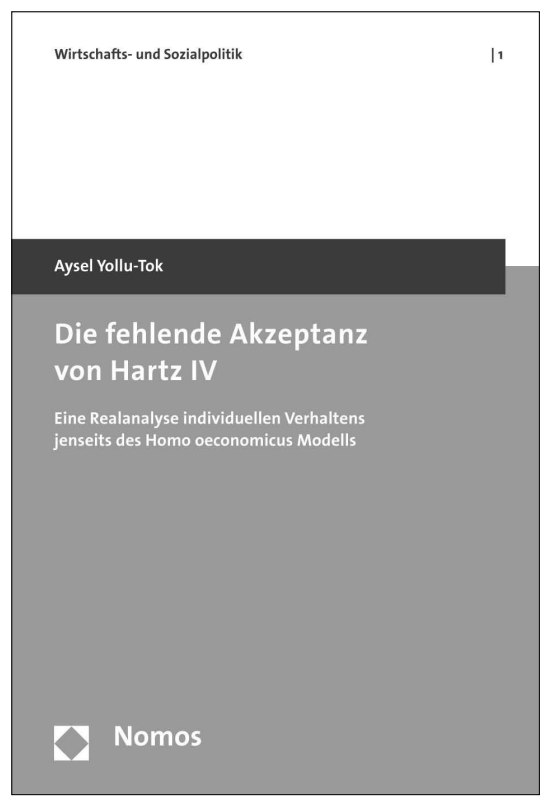

\author{
Die fehlende Akzeptanz \\ von Hartz IV \\ Eine Realanalyse \\ individuellen Verhaltens \\ jenseits des Homo \\ oeconomicus Modells \\ Von Dr. Aysel Yollu-Tok \\ 2010, 216 S., brosch., 29,- $€$, \\ ISBN 978-3-8329-5061-3 \\ (Wirtschafts- und Sozial- \\ politik, Bd. 1)
}

Die Hartz IV-Reform brach mit der Bismarckschen Tradition der sozialen Sicherung bei Erwerbslosigkeit. Dieser Bruch löste eine Welle von Demonstrationen aus, womit die fehlende Akzeptanz dieser Reform unterstrichen wurde. Auf welche Verhaltensmotive kann dieses Akzeptanzproblem zurückgeführt werden? Um dieser Frage nachzugehen, wird in der vorliegenden Arbeit das Modell des Homo oeconomicus um sozialpsychologische Erkenntnisse erweitert. Das neue Modell zeichnet sich vor allem dadurch aus, dass es nach eigennützigem Verhalten, aber auch nach weiteren Verhaltensmotiven fragen kann. Was war der Auslöser für die fehlende Akzeptanz der Grundsicherungsreform? Welchen Einfluss haben hierbei eigennützige Präferenzen? Inwieweit brach Hartz IV mit unterschiedlichen Gerechtigkeitsvorstellungen? Welchen Einfluss haben unterschiedliches Gewinn- und Verlustempfinden? Inwieweit spielt die Vorstellung, ob der Mensch erst durch Arbeit oder durch materielle Absicherung Teil der Gesellschaft ist, eine Rolle?

Der Erfolg einer Reform kann nicht nur auf der Basis objektiver Veränderungen bewertet werden, sondern muss gerade auch Individuen berücksichtigen, die sich mit ihrer subjektiven Einstellungen an das bisherige System angepasst haben

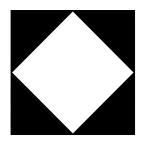

\section{Nomos}

